\title{
Influence des techniques de potabilisation sur la qualité radiologique de l'eau
}

\author{
J. REAL*, M. BOURRASSE*, J. FEUERSTEIN*, R. ROUXEL*
}

(Manuscrit reçu le 17 juin 1999, révisé le 30 août 1999, accepté le 9 octobre 1999)

RÉSUMÉ En cas de rejets radioactifs accidentels, l'eau potable ne représenterait pas la première préoccupation pour la définition du risque radiologique global ; cependant, elle constituerait le plus souvent la première inquiétude du consommateur. Les résultats présentés sont issus d'expérimentations réalisées en laboratoire et dont l'objectif était d'évaluer dans les mêmes conditions l'efficacité de deux filières de traitement d'eau potable sur l'élimination des radionucléides césium et strontium. Pour cela, une eau de captage a été contaminée avec des aérosols radioactifs représentatifs d'un accident. Puis, elle a été potabilisée d'une part, à l'aide d'une filière classique (clarification et filtration sur sable puis sur charbon actif), et d'autre part, par une technique moins classique mais facilement intégrable au sein d'une filière déjà existante, dans laquelle le sable est remplacé par de la zéolite. Le rendement à la fin du traitement utilisant le sable est moyen pour le césium (de 67 à $73 \%$ selon le type de coagulant injecté) et devient franchement médiocre pour le strontium (entre 46 et $51 \%$ ). Les résultats obtenus avec la filière comprenant de la zéolite sont très satisfaisants. Les rendements en fin de traitement atteignent $99 \%$ aussi bien pour le strontium que pour le césium et ce, quelque soit le type de coagulant utilisé. La présente étude a donc permis de démontrer la faible efficacité des traitements dits «classiques» d'eau potable face à l'élimination des radionucléides césium et strontium, mais aussi d'esquisser les traits d'une nouvelle filière encore à perfectionner de décontamination radioactive de l'eau.

ABSTRACT Influence techniques of potabilisation on the radiological quality of water.

In case of accidental radioactive discharges, the drinking water would not represent the first concern for the definition of the total radiological risk; however, it would generally constitute the first concern of the consumer. The purpose of the results come from experiments carried out in laboratory and were to evaluate the efficiency of two type of processing of drinking water on the elimination of the radionuclides cesium and strontium. Therefore, a catchment water was contaminated with radioactive aerosols representative of an accident. Then, it was potabilited on the one hand, using a traditional process (clarification and filtration on sand then on activated carbon), and on the other hand, by a less traditional but easily integrable technique within an already existing process, in which sand is replaced by zeolite. The performance at the end of the processing using sand is average for cesium (from 67 to $73 \%$ according to the type of coagulant injected) and becomes frankly poor for strontium (between 46 and $51 \%$ ). The results obtained with the process including of zeolite are very satisfactory. The performance at the

IPSN, Institut de protection et de sûreté nucléaire, département de protection de l'environnement, service d'études et de recherche, C.E. Caradache, 13108 Saint-Paul-Lez-Durance Cedex, France. 
end of the processing as well reach $99 \%$ for strontium as for cesium whatever the type of coagulant used. The present study thus made it possible to show the insufficiency of the processing known as "traditional" of drinking water towards to the elimination of the radionuclides cesium and strontium, but also to outline the features of a new process still to improve radioactive decontamination of water.

\section{Introduction}

Les études consacrées à l'efficacité des traitements de potabilisation sur la réduction de la radioactivité dans l'eau sont peu nombreuses. Certaines ont été réalisées en usine (Masschelein et al., 1989, à Taifler en Belgique), sur des pilotes (Pineira et al., 1989 sur de l'eau de la Seine), ou bien en laboratoire (Colle 1985; Montiel et al., 1991 sur des eaux de la Seine). Dans tous les cas, les résultats sont très disparates et difficilement comparables car les eaux étudiées ont des origines et donc des caractéristiques physico-chimiques différentes et les conditions expérimentales sont souvent mal connues. Il semble cependant que la coagulation-floculation-décantation (traitement le plus utilisé) élimine au maximum $50 \%$ des radionucléides présents dans l'eau. La filtration lente sur sable permettrait d'éliminer $60 \%$ du strontium mais un phénomène de relargage est inévitable. La filtration sur charbon actif est inefficace ainsi que la désinfection.

Devant la diversité de ces résultats, de nouvelles expérimentations ont été réalisées avec le souci de se placer dans des conditions les plus proches possibles des usines de traitement d'eau potable. Dans ce cadre, deux filières de traitement de potabilisation ont été expérimentées sur une eau de captage. Elles comprennent, dans les deux cas, une coagulation-floculation-décantation, suivie pour la première, d'une filtration sur sable et pour la deuxième, d'une filtration sur zéolite. Elles se terminent toutes les deux par une filtration sur charbon actif.

\section{Matériel et méthodes}

Deux voies expérimentales ont été suivies. La première a consisté à réaliser des essais statiques en flacons afin d'étudier les échanges entre les différentes phases et la deuxième a permis grâce à des essais en colonne de déterminer quantitativement la rétention des éléments radioactifs par l'ensemble des matériaux constitutifs de la filière.

L'eau utilisée pour les essais provient du captage d'eau potable d'Anthon situé

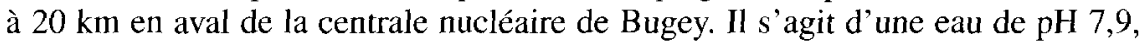
ses caractéristiques physico-chimiques permettent de la considérer légèrement incrustante. 


\section{TABLEAU I}

Caractéristiques physiques des matériaux.

Physical characteristics of materials.

\begin{tabular}{|c|c|c|c|}
\hline & sable & zéolite & charbon actif \\
\hline granulométrie en mm & 0,63 à 1,25 & 0,63 à 1,25 & 0,4 à 1,7 \\
\hline taille effective (TE) en mm & 0,63 & 0,63 & 0,6 \\
coefficient d'uniformité (CU) & 1,58 & 1,6 & 1,8 \\
surface spécifique & non communiqué & $400 \mathrm{~m}^{2} \mathrm{~cm}^{-3}$ & $1200 \mathrm{~m}^{2} \mathrm{~g}^{-1}$ \\
\hline masse volumique g cm & non communiqué & 1,71 à 2,16 & 0,55 \\
\hline
\end{tabular}

Les radionucléides césium et strontium sont déposés par gravité dans l'eau sous forme d'aérosols produits par l'installation POLYR (Camarasa-Claret et al., 1997) qui est un dispositif expérimental pouvant générer des aérosols se rapprochant de ceux produits dans l'enceinte du réacteur nucléaire lors d'un accident avec rejet atmosphérique. Ces aérosols sont composés de 14 éléménts dont 2 radioactifs, le ${ }^{134}$ Cs et le ${ }^{85} \mathrm{Sr}$. Les activités moyennes de l'eau après contamination ont été mesurées par spectrométrie $\gamma$ (Eurisys Mesure détecteur germanium hyperpur type $\mathrm{N}$ -mesures sur 4096 canaux) et sont de $26842 \pm 260 \mathrm{~Bq} \mathrm{l}^{-1}$ soit $0,63 \mathrm{ng} \mathrm{l}^{-1}$ pour ${ }^{134} \mathrm{Cs}$ et $32587 \pm 320 \mathrm{~Bq} \mathrm{l}^{-1}$ soit $0,03 \mathrm{ng} \mathrm{l}^{-1}$ pour ${ }^{85} \mathrm{Sr}$. Les éléments stables sont présents aux concentrations de $13,3 \mu \mathrm{g} \mathrm{l}^{-1}$ pour le césium et $11,5 \mu \mathrm{g} \mathrm{l}^{-1}$ pour le strontium.

Les matériaux utilisés (Tab. I) proviennent tous de sociétés fournissant les professionnels du traitement de l'eau.

Le sable quartzeux est le matériau de base pour la plupart des filtres actuels. Ce sable, appelé quartzite de filtration 8/12 provient de la société Silice et Kaolin.

La clinoptilolite (zéolite) provient de la SO.M.E.Z. Sa granulomètrie était de 1 à $5 \mathrm{~mm}$. Afin de se placer dans les mêmes conditions expérimentales, nous avons dû la broyer et la tamiser par voie humide pour obtenir une granulomètrie de 0,63 à $1,25 \mathrm{~mm}$ et éliminer les nombreuses particules fines obtenues lors du broyage.

Le charbon actif provient de la société CECA /ATO, filiale de Elf Atochem. Le produit " Acticarbone NC 35 0,4/1,7 mm " est un charbon de coques de noix de coco activé physiquement à la vapeur.

\subsection{Les essais statiques en flacons ou " batchs"}

Deux types d'essais statiques ont été effectués. Des essais de coagulation ou “ JarTest ", qui ont pour but de rechercher la nature et les doses de réactifs permettant 
de traiter une eau dans les meilleures conditions de potabilisation, et des essais d'adsorption sur charbon actif et zéolite, nécessaires pour déterminer les isothermes d'adsorption des éléments étudiés.

\section{Coagulation}

Deux réactifs utilisés couramment dans les stations de traitement de l'eau et commercialisés par Elf Atochem ont été testés: 5 et 8 ;

- le chlorure ferrique à $41 \%$; il est utilisé dans la gamme de $\mathrm{pH}$ compris entre

- le WAC-HB est un polychlorure d'aluminium, il est utilisé dans une plage de pH entre 5 et 10 .

Ces essais ont été effectués en agitant simultanément l'eau contenue dans les six postes d'un floculateur. Les vitesses de rotation de l'agitateur étaient de $250 \mathrm{tr} / \mathrm{min}$ pendant 2 minutes puis de $40 \mathrm{tr} / \mathrm{min}$ en maintenant une température de $17^{\circ} \mathrm{C}$ (Degrémont, 1989). L'influence de la quantité de coagulant a été étudiée par l'ajout de doses croissantes dans la série des six béchers. L'activité résiduelle en césium et strontium a été mesurée dans chaque flacon. Ce test permet aussi de déterminer la dose optimale à introduire pour les essais en colonne.

Les quantités de coagulant ont été déterminées à partir des doses usuelles, c'està-dire de 0 à $50 \mathrm{ml} \mathrm{m}^{-3}$ (Capellier, 1992). Le $\mathrm{pH}$ optimum a été obtenu en introduisant des doses croissantes de $\mathrm{NaOH}$ à $0,1 \mathrm{~mol}^{-1}$ dans la série de béchers.

\section{Adsorption}

Le floculateur a également été utilisé pour déterminer l'adsorption des radionucléides sur la zéolite et le charbon actif. Pour cela, des quantités croissantes de charbon actif et de zéolite ont été introduites sous forme de poudre dans la série des six béchers. La réduction en poudre du matériau ainsi que l'agitation du contenu des six béchers permet d'atteindre l'équilibre plus rapidement.

TABLEAU II

Filières de traitement d'eau expérimentées.

Types of processing of water tested.

\begin{tabular}{|l|l|}
\hline \multicolumn{1}{|c|}{ filière 1 } & \multicolumn{1}{c|}{ filière 2} \\
\hline coagulation-floculation-décantation, & coagulation-floculation-décantation, \\
filtration sur sable, & filtration sur zéolite, \\
\hline filtration sur charbon actif. & filtration sur charbon actif. \\
\hline
\end{tabular}




\subsection{Les essais dynamiques en colonne}

Des conditions identiques à celles que l'on trouve dans les stations de traitement d'eau potable ont été respectées, en particulier en ce qui concerne les temps de contact, les vitesses de filtration et la granulométrie des matériaux utilisés. La rétention et le relargage des radionucléides ont été évalués.

Deux filières de traitement ont été simulées comme le montre le tableau II.

La colonne est constituée d'un tube en verre de $1 \mathrm{~m}$ de haut et de $5 \mathrm{~cm}$ de diamètre intérieur, elle est remplie sur une hauteur de $40 \mathrm{~cm}$ de matériaux (sable, zéolite ou charbon actif). Une vitesse de filtration de $5 \mathrm{~m} \mathrm{~h}^{-1}$ et un débit de $101 \mathrm{~h}^{-1}$ ont été appliqués pour réaliser une filtration gravitaire.

L'eau, après avoir été contaminée, est soumise à une coagulation-floculationdécantation dans un agitateur à hélice afin de respecter les procédures habituelles. Elle est ensuite introduite dans la colonne de sable ou de zéolite puis dans celle contenant le charbon actif. Durant cette étape des prélèvements d'eau sont réalisées toutes les $15 \mathrm{~min}$ en bas de chaque colonne et sont mesurés en spectrométrie $\gamma$ afin d'évaluer la rétention des radionucléides sur le matériau.

Le relargage est évaluée en remplaçant à la fin de la séquence précédente l'eau contaminée par de l'eau naturelle.

\section{Résultats et discussion}

\subsection{Essais statiques en flacons ou «batch»}

\section{Jar-test}

La figure 1 montre que le profil de la rétention des radioéléments n'est influencé, ni par le type de coagulant, ni par sa concentration (entre 0 et $50 \mathrm{mg} \mathrm{l}^{-1}$ ). La fixation du césium est négligeable puisque elle est de l'orde de $1 \%$. Ces résultats sont cohérents avec nos connaissances sur cette technique et sur le comportement du césium. En effet, la coagulation-floculation élimine les matières en suspension contenues dans l'eau, or le césium est surtout lié à la phase soluble et non particulaire; il est donc peu concerné par ce traitement. La rétention du strontium est de $10 \%$ et peut avoir deux origines, soit une adsorption sur les hydroxydes de fer et d'aluminium provenant des adjuvants de coagulation (Montiel et al., 1991), soit une précipitation du strontium avec les éléments naturels de l'eau et en particulier les sulfates et les carbonates (Nilson et al., 1985). Les résultats de ces essais, en tenant compte de la grosseur des flocs, du $\mathrm{pH}$ et de l'aspect du surnageant, ont permis de 
déterminer les doses de soude et de coagulant à introduire lors des essais en colonne. Ces doses sont de $30 \mathrm{ml} \mathrm{l}^{-1}$ de $\mathrm{FeCl}_{3}$ et de WAC-HB et respectivement 6 et $8 \mathrm{mll}^{-1}$ de $\mathrm{NaOH}$ pour les essais avec $\mathrm{FeCl}_{3}$ et WAC-HB.

\section{Avec le chlorure ferrique}
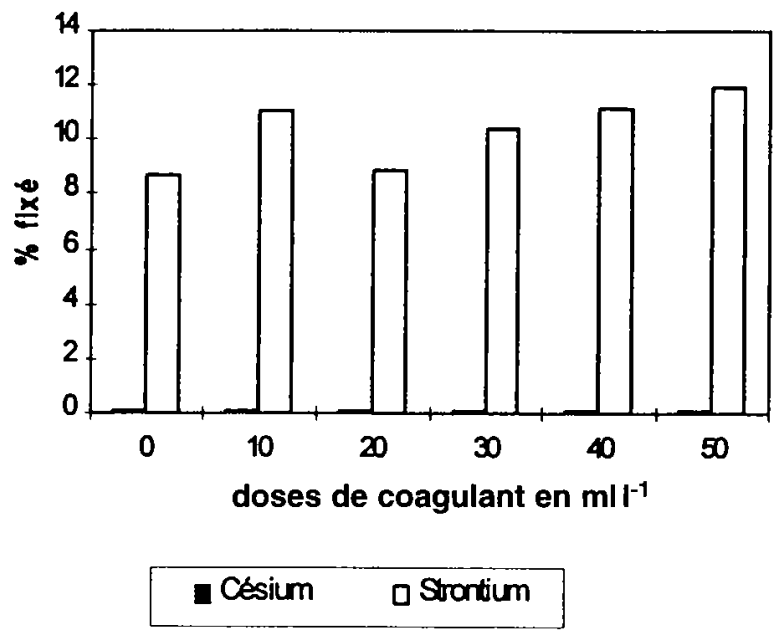

Avec le WAC-HB

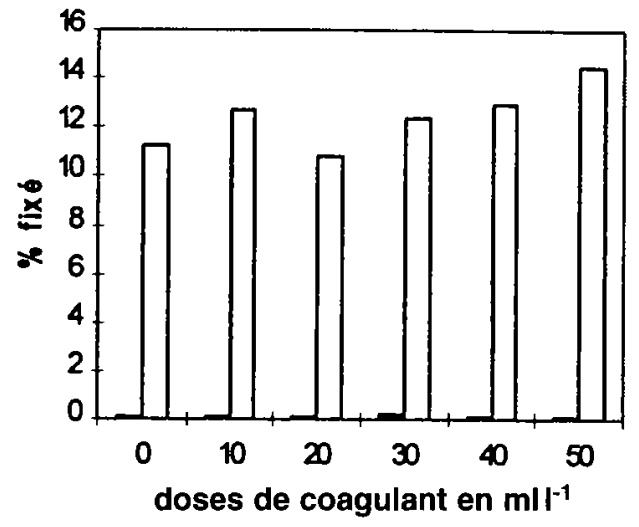

\section{- Césium a Srontium}

Fig. 1. - Rétention du césium et du strontium en fonction de doses croissantes de coagulants. Retention of caesium and strontium for increasing amounts of coagulant. 


\section{Adsorption}

- La clinoptilolite

La capacité d'échange du césium et du strontium avec la clinoptilolite a été testée par ajout de quantités croissantes de ce matériau. L'isotherme d'adsorption présenté sur la figure 2 a été tracé à partir des résultats de mesure selon l'équation de Freundlich :

$$
X / m=k C^{n}
$$

où $X$ est l'activité adsorbée en $\mathrm{Bq}, m$ est la masse de clinoptilolite en $\mathrm{g}$. $C$ est l'activité volumique finale en $\mathrm{Bq}^{-1}, k$ et $n$ sont des constantes.

Étant donné le peu de strontium échangé, nous n'avons pas pu tracer l'isotherme d'échange. Le fait que le césium se soit fixé en plus grande quantité que le strontium peut s'expliquer par une sélectivité dans l'échange. De nombreux facteurs interviennent dans cette sélectivité et en particulier, la valence et le volume des ions, leur degré d'hydratation, les dimensions des pores, la proximité des sites d'échange. Il a été montré (Régis, 1989) que les échanges suivaient l'ordre suivant : $\mathrm{Cs}^{+}>\mathrm{K}^{+}$ $>\mathrm{NH}_{4}^{+}>\mathrm{Na}^{+}>\mathrm{Ba}^{2+}>\mathrm{Sr}^{2+}$. Cette hiérarchie pourrait expliquer les résultats que nous avons trouvé.

La capacité d'échange de la zéolite a aussi été évaluée en mesurant les pourcentages de césium et de strontium échangés en fonction de quantités croissantes de matériau (Fig. 3). Pour le césium, on constate que pour un contact d'une heure,

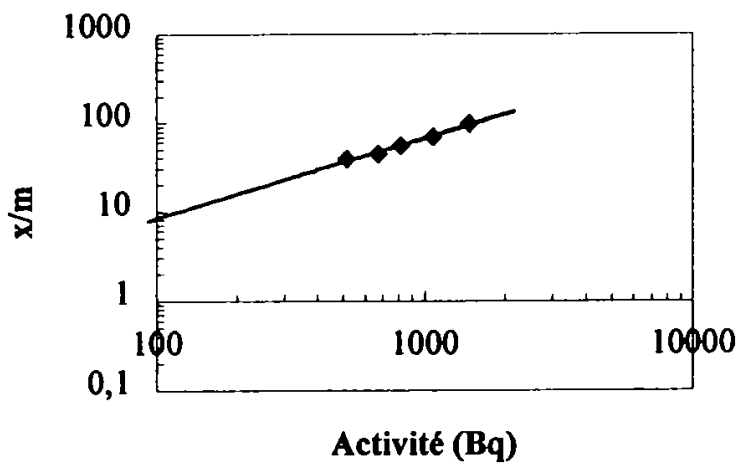

Fig. 2. - Isotherme d'échange du césium sur la clinoptilolite pendant un contact de une heure. Isotherm of exchange of cesium on the clinoptilolite during a one hour contact. 


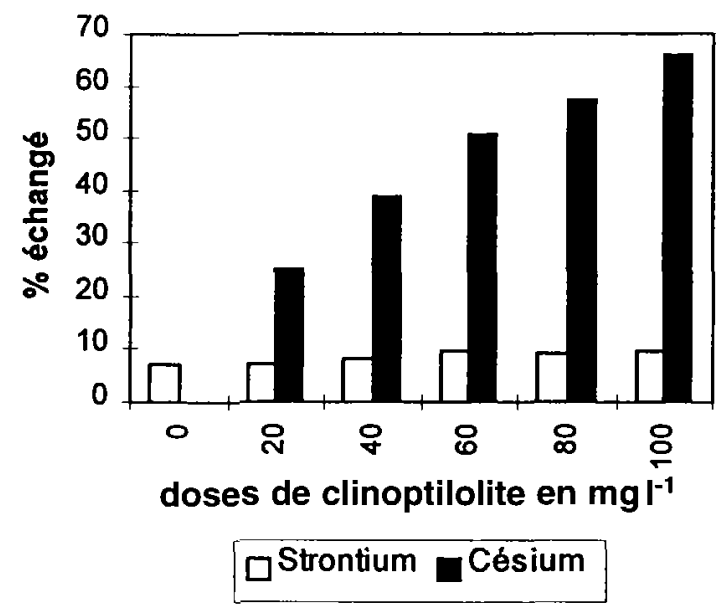

Fig. 3. - Adsorption du césium et du strontium sur la clinoptilolite pendant un contact de une heure et pour des doses croissantes de clinoptilolite.

Adsorption of caesium and strontium on the clinopilolite during a one hour contact and for increasing amounts of clinoptilolite.

plus la masse de zéolite augmente, plus le pourcentage de césium échangé est grand. Ce résultat serait intéressant pour traiter le cas d'une pollution ponctuelle ; la zéolite pourrait être mise en œuvre de la même manière que le charbon actif en poudre. Un poste de coagulation-floculation-décantation suffirait alors pour traiter l'eau.

Les résultats concernant le strontium montrent que l'adsorption sur la zéolite est quasi indépendante de la quantité de matériau en jeu.

\section{- Le charbon actif}

Le taux d'adsorption du césium sur le charbon actif est très faible, celui du strontium est voisin de $10 \%$ avec un profil de rétention identique à celui des autres procédés de traitement étudiés.

Ces résultats sont en accord avec les connaissances sur le comportement du charbon actif qui est essentiellement utilisé en traitement des eaux potables pour l'élimination de micropolluants organiques et pour neutraliser le chlore (Capellier, 1992). 
avec le $\mathrm{FeCl}_{3}$

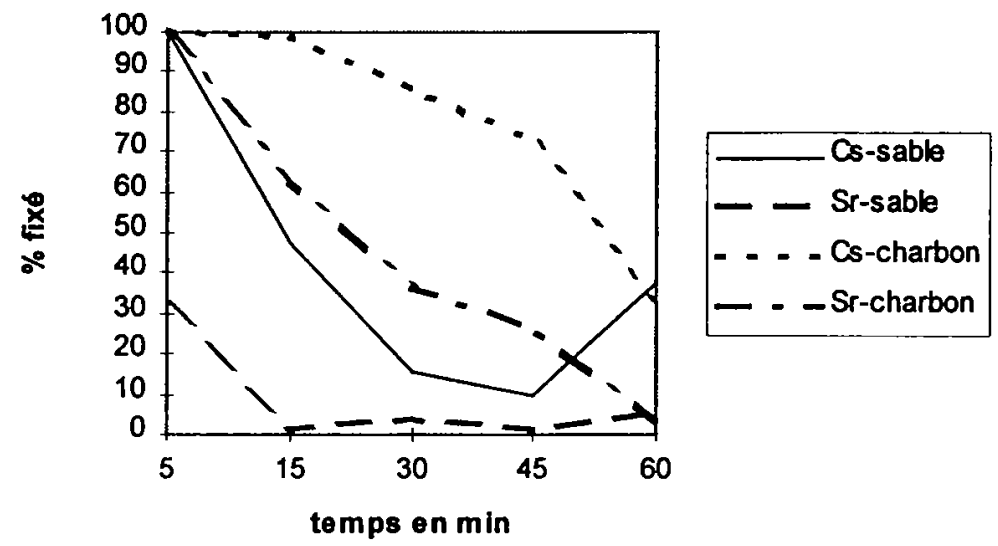

avec le WAC-HB

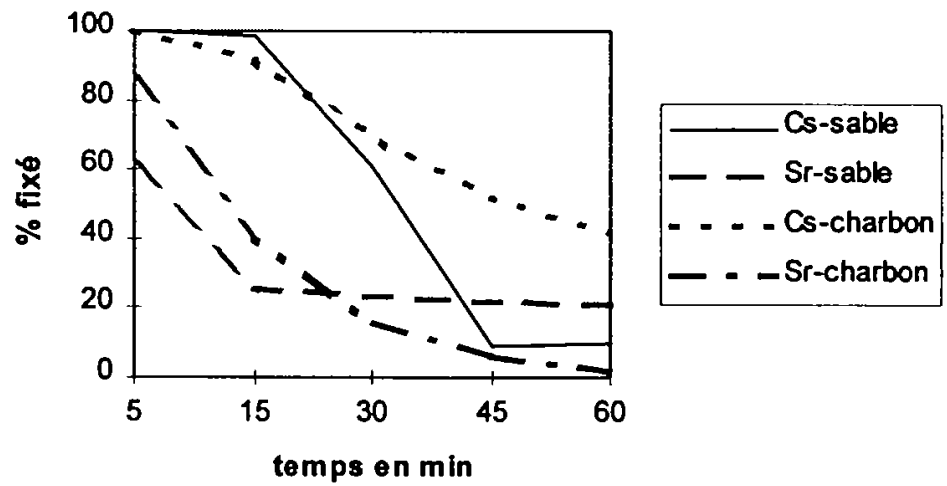

Fig. 4. - Rétention par filtration sur sable et charbon actif en fonction du temps.

Retention by filtration on sand and activated carbon according to time. 


\subsection{Les essais en colonne}

La rétention puis le relargage des radionucléides ont été évalués à la fin de chaque processus élémentaire en considérant les traitements placés en amont (coagulation-floculation avec le WAC-HB ou $\mathrm{FeCl}_{3}$ ). L'efficacité globale de chaque filière a ensuite été déterminée.

Dans tous les cas, les résultats ont été exprimés en pourcentages d'activité fixée ou relarguée par rapport à l'activité initiale et ce, en fonction des temps de prélèvement.

\section{- La filière 1}

La rétention du césium sur le sable et sur le charbon actif présente un profil similaire pour les deux coagulants utilisés (Fig. 4) si on fait abstraction du taux de fixation du sable à 60 minutes avec le $\mathrm{FeCl}_{3}$. La fixation est de $100 \%$ au bout de 5 minutes et décroit sans palier pour atteindre à 60 minutes $40 \%$ pour le charbon et $10 \%$ pour le sable.

La rétention du strontium sur le charbon est identique pour les 2 coagulants avec diminution progressive de $90-100 \%$ à $2-5 \%$ lorsque l'on passe de 5 à 60 minutes. Par contre la fixation du strontium sur le sable décroît avec présence d'un palier à 15 minutes pour les deux coagulants, mais elle est deux fois plus importante dans le cas du WAC-HB.

Les résultats du relargage (Fig. 5) montent que lorsque on utilise le $\mathrm{FeCl}_{3}$, le filtre à sable relargue très peu de strontium contrairement au césium. Ces résultats sont en accord avec ceux de Montiel qui a constaté que, dès l'arrêt de l'ajout de strontium, aucun relargage n'a été observé. Ceci pourrait confirmer son hypothèse disant que le strontium est adsorbé sur l'hydroxyde ferrique retenu par le sable lors de la filtration et que cette adsorption est stable. Dans le cas du WAC-HB, le relargage du strontium par le sable est immédiat, mais passe en 15 minutes de $25 \%$ à une valeur quasi nulle. La cinétique de relargage du césium par le filtre à sable est identique pour les deux coagulants avec des valeurs de 20 à $15 \%$ au départ et $5 \%$ au bout de 15 minutes suivies d'un palier.

Les profils de relargage du strontium par le charbon actif sont très semblables quel que soit le coagulant utilisé avec élimination de $17-20 \%$ à $5 \%$ de 5 à 60 minutes. Le relargage du cesium par le charbon se fait de façon continue autour de $10 \%$ pour le $\mathrm{FeCl}_{3}$ et $5 \%$ pour le WAC-HB.

\section{- La filière 2}

La rétention des radionucléides par la zéolite est immédiate et quasi-complète. Ceci est surprenant pour le strontium car lors des essais d'échange sur la zéolite, nous 


\section{avec le $\mathrm{FeCl}_{3}$}

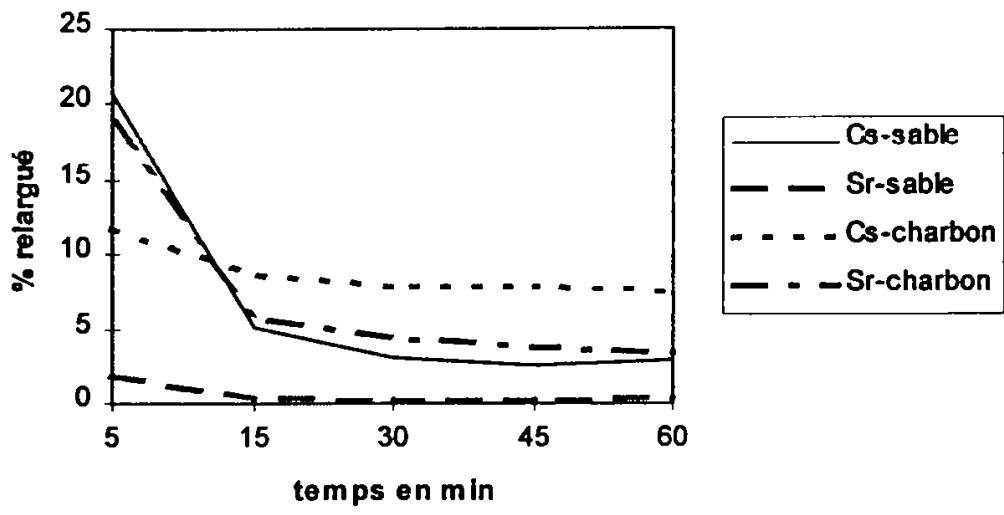

avec le WAC-HB
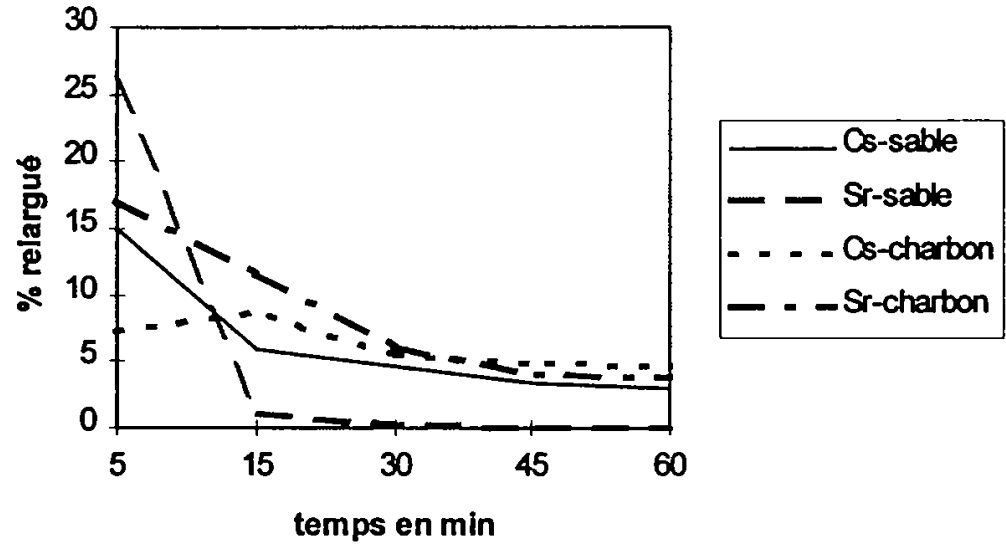

Fig. 5. - Relargage au niveau du filtre à sable et du charbon actif en fonction du temps.

Salting out on the level of the sand filter and the activated carbon according to time. 
avions constaté une faible fixation. Au cours du temps, la rétention atteint $99 \%$ pour les deux radionucléides quelque soit le coagulant utilisé. Les résultats issus de la bibliographie donnaient des valeurs similaires mais pour des expériences dans lesquelles les vitesses de filtration étaient de l'ordre du $\mathrm{m} \mathrm{j}^{-1}$. Ici, nous avons utilisé une vitesse de $5 \mathrm{~m} \mathrm{~h}^{-1}$, il semble donc que la vitesse de filtration n' ait pas d'influence sur la rétention du césium et du strontium. Au vu de ces bons résultats, la filtration sur charbon actif semble peu utile. Le relargage du césium par la zéolite lors de l'arrêt de la pollution est très faible et en dessous du seuil de détection quel que soit le coagulant utilisé. Pour le strontium, il est inférieur à $0,1 \%$ et est plus important dans le cas du $\mathrm{FeCl}_{3}$ contrairement à ce que l'on observait dans le cas de la filière 1. À la fin de l'expérience, ce relargage est toujours constant. Dans le cas du WAC-HB, le relargage est très faible mais constant.

La quantité adsorbée lors de la filtration sur charbon actif étant très faible, la désorption lors de l'arrêt de la pollution est très faible et en dessous du seuil de détection.

\section{- Bilan de l'efficacité des deux filières}

Les résultats montrant l'efficacité de chaque filière sont présentés dans le tableau III sous forme de pourcentages de césium ou de strontium retenus à la fin de chaque procédé par rapport à la quantité présente au début du procédé. L'efficacité totale de la filière est présentée dans la dernière ligne, elle correspond à la quantité retenue après la succession des différents procédés.

Dans le cas de la filière 1 , la rétention totale du césium est de 67 et de $73 \%$, respectivement, lors de l'utilisation du $\mathrm{FeCl}_{3}$ et du WAC-HB. Compte tenu des marges d'erreur, le fait d'utiliser du $\mathrm{FeCl}_{3}$ ou du WAC-HB ne change pas l'efficacité de cette filière. Le pouvoir de rétention global de la filière 1 est tout de même convenable ; par contre, des problèmes de relargage apparaissent lorsque la pollution s'arrête.

En comparant nos résultats avec ceux trouvés dans la littérature, nous constatons des différences notables. La rétention des radionucléides lors de la coagulation-floculation-décantation est en général plus faible que celle obtenue par Colle ou Pineira. En ce qui concerne la filtration sur sable, nous obtenons des résultats plus faibles que Pineira dans le cas de l'utilisation du chlorure ferrique et plus élevés dans le cas de l'utilisation du WAC-HB. Ces différences peuvent s'expliquer par le fait que nous avons utilisé une eau d'origine souterraine alors que Colle et Pineira ont utilisé l'eau de Seine, beaucoup plus chargée en M.E.S. et que d'autre part les conditions d'expérimentation étaient différentes. La filtration sur charbon actif nous donne des résultats foncièrement différents de ceux obtenus par Pineira (99\%), nos résultats semblent cependant plus en accord avec les caractéristiques spécifiques du charbon actif. 
TABLEAU III

Pouvoir de rétention des deux filières en \% fixé.

Retention capacity of two process in $\%$ fixed.

\begin{tabular}{|c|c|c|c|c|c|c|c|c|}
\hline & \multicolumn{4}{|c|}{ Filière 1} & \multicolumn{4}{|c|}{ Filière 2} \\
\hline & \multicolumn{2}{|c|}{ césium } & \multicolumn{2}{|c|}{ strontium } & \multicolumn{2}{|c|}{ césium } & \multicolumn{2}{|c|}{ strontium } \\
\hline & $\mathrm{FeCl}_{3}$ & WAC-HB & $\mathrm{FeCl}_{3}$ & WAC-HB & $\mathrm{FeCl}_{3}$ & WAC-HB & $\mathrm{FeCl}_{3}$ & WAC-HB \\
\hline $\begin{array}{l}\text { Coagulation- } \\
\text { floculation- } \\
\text { décantation }\end{array}$ & $2 \pm 0.1$ & $4 \pm 0.3$ & $13 \pm 0.9$ & $14 \pm 1$ & $4 \pm 0.3$ & $4 \pm 0.3$ & $26 \pm 1,8$ & $8 \pm 0.6$ \\
\hline $\begin{array}{l}\text { Filtration } \\
\text { sur sable }\end{array}$ & $28 \pm 2$ & $52 \pm 2.6$ & $11 \pm 0.8$ & $27 \pm 1.9$ & & & & \\
\hline $\begin{array}{l}\text { Filtration } \\
\text { sur zéolite }\end{array}$ & & & & & $99 \pm 6.9$ & $99 \pm 6.9$ & $99 \pm 6.9$ & $99 \pm 6.9$ \\
\hline $\begin{array}{l}\text { Filtration sur } \\
\text { charbon actif }\end{array}$ & $54 \pm 3.8$ & $40 \pm 1.6$ & $37 \pm 3$ & $14 \pm 0.9$ & $25 \pm 7$ & $78 \pm 15.6$ & $40 \pm 11.2$ & $75 \pm 48.8$ \\
\hline $\begin{array}{l}\text { succession des } \\
3 \text { procédés }\end{array}$ & $67 \pm 4.7$ & $73 \pm 5.1$ & $\mathbf{5 1} \pm 7.5$ & $46 \pm 3.2$ & $99 \pm 6.9$ & $99 \pm 6.9$ & $99 \pm 6.9$ & $99 \pm 6.9$ \\
\hline
\end{tabular}

Dans le cas de la filière 2, les résultats de rétention du césium et du strontium sont plus prometteurs que ce que nous attendions au vu des essais d'échange sur la zéolite puisque l'efficacité totale est de $99 \%$ pour le césium et le strontium quel que soit le coagulant choisi.

\section{Conclusion}

Deux filières de traitement d'eau potable ont été testées sur une eau souterraine pour étudier leur efficacité vis-à-vis de la rétention des radionucléides césium et strontium. La première est classique, elle se compose d'une coagulation-floculation-décantation, suivie d'une filtration sur sable puis sur charbon actif. La seconde est plus originale, mais de mise en œuvre aisée, puisque il suffit de remplacer la filtration sur sable par une filtration sur zéolite. Chaque procédé est alors étudié en fonction de ceux placés en amont.

La phase de coagulation-floculation-décantation ne s'est pas avérée très efficace puisque seulement 2 à $4 \%$ de césium et 8 à $26 \%$ de strontium ont été éliminés. Cependant, la faible teneur en matières en suspension de l'eau utilisée était un facteur limitant pour cette méthode et son efficacité sur un autre type d'eau pourrait être meilleure. Le coagulant à base de $\mathrm{FeCl}_{3}$ semble plus efficace pour éliminer le strontium alors que la rétention du césium est la même pour les deux coagulants. 
Le rendement à la fin de la filière classique est moyen pour le césium (de 67 à $73 \%$ selon le type de coagulant injecté) et devient médiocre pour le strontium (entre 46 et $51 \%$ ).

Les résultats de la filière incluant la zéolite sont par contre excellents. Les rendements atteignent $99 \%$ aussi bien pour le strontium que pour le césium, et ce quel que soit le type de coagulant utilisé. Cette filière semble donc un bon moyen pour éliminer les radionucléides de l'eau. Cependant, pour véritablement conclure sur l'intérêt de cette filière, il serait bon de réaliser des intercomparaisons entre différents indicateurs tels que son efficacité en fonction de différents types d'eau (de surface, souterraine...), les difficultés opérationelles de mise en œuvre au sein d'usines et leur coût, les effets sur la qualité de l'eau en terme de potabilité ainsi que la gestion des déchets.

Remerciements : Les travaux présentés ont été partiellement financés par EDF et Euratom dans la cadre d'accords de coopération scientifique.

\section{RÉFÉRENCES}

Camarasa-Claret C., Persin F., Real J. (1997) Dissolution du césium et du strontium contenus dans des aérosols radioactifs accidentels. Actes du $13^{e}$ congrès français sur les aérosols, 2-3 décembre 1997, Paris, pp. 25-31.

Colle C. (1985) Définition des coefficients de distribution $\left(K_{d}\right)$ au niveau des stations de traitement d'eau potable situées sur la Seine en aval du site de Nogent-sur-Seine. SERE/LRT/VEN $1665-$ $04,1985,29 \mathrm{p}$.

Capellier M. (1992) Traitement d'eau potable. E.N.G.E.E.S.-S.A.U.R., 1992, 11 chapitres.

Degrémont (1989) Mémento technique de l'eau. Lavoisier-Paris, 1989, 1459 p.

Masschelein W.J., Goossens R., Delville A. (1989) Impact of fall-out and management of removal by conventional water treatment. Water resource consequences following a nuclear event, pp. 139 151. The university of Strathclyde, Glasgow, septembre 1989.

Montiel A., Welté B., Franchet C., Legrand S. (1991) Étude de l'élimination du strontium dans les filières de traitement de potabilisation des eaux de surface. Environmental Technology 12, 797-802.

Nilson K., Skytte Jensen B., Carlsen L. (1985) The migration chemistry of strontium. Topical report. Work performed in the frame of R\&D Programme. Management and disposal of radioactive waste, $n^{\circ}$ 194-81-6 WASDK pp. 187-188.

Pineira T., Fauchere L. (1989) Élimination des éléments radioactifs dans une filière d'eau potable. Résultats des essais réalisés sur le pilote installé au CEN-Saclay. DCENS/SPR/89-371, décembre $1989,10 \mathrm{p}$.

Régis R. (1989) Application des zéolites naturelles au traitement des eaux. L'eau, l'industrie et les nuisances $\mathrm{n}^{\circ} 129,43-46$.

Rocher P. (1990) Les zéolites naturelles, Des matériaux d'avenir, pour combien de temps encore ? Géochronique $\mathrm{n}^{\circ} 34,13-17$. 